\title{
On the Dimension of a Face Exposed by Proper Separation of Convex Polyhedra
}

\author{
Stephen E. Wright
}

Received: 27 June 2008 / Revised: 13 September 2008 / Accepted: 18 September 2008 /

Published online: 7 October 2008

(C) Springer Science+Business Media, LLC 2008

\begin{abstract}
Whenever two nonempty convex polyhedra can be properly separated, a separating hyperplane may be chosen to contain a face of either polyhedron. It is demonstrated that, in fact, one or the other of the polyhedra admits such an exposed face having dimension no smaller than approximately half the larger dimension of the two polyhedra. An example shows that the bound on face dimension is optimal, and a linear programming representation of the problem is given.
\end{abstract}

Keywords Linear programming · Calculation of supporting hyperplanes · Detecting set intersection $\cdot$ Polyhedral relative interiors

\section{Introduction}

Separation of sets plays a fundamental role in many areas of mathematics. Familiar classical results relate the quality of linear separation of two convex sets in a real vector space to the degree to which the two sets fail to overlap. A typical result along these lines is the following [4, Theorem 11.3]:

Two nonempty convex sets in a Euclidean space can be properly separated by some hyperplane if, and only if, their relative interiors are disjoint.

Proper separation means that at least one of the two convex sets does not lie entirely within the separating hyperplane. In particular, separation of two sets is always proper when either set has nonempty interior.

Often, the separating hyperplane can be chosen to support the closure of one or the other of the two sets, in which case the intersection of the hyperplane with that closure is an exposed face of the closure. Such a face will be a singleton when the supported

S.E. Wright $(\varangle)$

Department of Mathematics and Statistics, Miami University, Oxford, OH 45056, USA

e-mail:wrightse@muohio.edu 
closure is rotund (that is, strictly convex), but one generally expects a non-singleton face when the separated sets have polyhedral closures, particularly if the polyhedra are themselves disjoint. However, it may not be obvious that a non-singleton face is always possible when, for example, the polyhedral boundaries meet only at a common vertex. This motivates the following result.

Theorem 1 Let d denote the larger dimension of two nonempty convex polyhedra in a Euclidean space. If the polyhedra can be properly separated, then one or the other has a face of dimension at least $\lfloor d / 2\rfloor$ contained in a properly separating hyperplane.

This result has potential value for computation. For instance, an immediate corollary is that two polygons in the plane have nonintersecting relative interiors if, and only if, they lie in the opposite closed halfplanes determined by a line containing an edge of one or the other of the two polygons. This criterion can be implemented efficiently to detect the overlap of unpreprocessed convex hulls of finite point-sets; alternatively, the criterion can be vectorized to detect overlaps among a large collection of (say) triangles or regular polygons. Analogous tools for higher dimensions might likewise be based on such a criterion, or perhaps on Theorem 2 below. The author's interest in detecting the intersection of polyhedral relative interiors arises in connection with problems on tiling and packing, an application in which intersecting boundaries are generally expected and strong separation is unlikely.

Knowledge and identification of the minimal supporting face dimension may also be of value in data-mining methods and applications (for example, see [1, 2]).

A short proof of Theorem 1 is given in the next section, while in Sect. 3 we present examples showing that the lower bound $\lfloor d / 2\rfloor$ is optimal for all $d$. The final section indicates how such a separating hyperplane can be calculated by linear programming methods in polynomial time (for rational input data) in the case where one of the polyhedra is known to have nonempty interior.

\section{Proof of Theorem 1}

Our proof uses standard concepts from the theory of convex polyhedra, as presented in $[4,5]$. The two main facts needed are that the Minkowski difference of convex polyhedra is itself a convex polyhedron, and the fact that two convex sets can be properly separated from each other if, and only if, their Minkowski difference can be properly separated from the origin.

Consider properly separated nonempty polyhedra $\mathcal{A}$ and $\mathcal{B}$, and let $n=$ $\operatorname{dim}(\mathcal{A}-\mathcal{B})$. First, we demonstrate the existence of a hyperplane $\mathcal{H}$ properly separating the origin from $\mathcal{A}-\mathcal{B}$, such that the face $\mathcal{F}=\mathcal{H} \cap(\mathcal{A}-\mathcal{B})$ of $\mathcal{A}-\mathcal{B}$ satisfies $\operatorname{dim} \mathcal{F} \geq n-1$. Note that if the origin does not lie in the affine hull of $\mathcal{A}-\mathcal{B}$, then there exists a hyperplane $\mathcal{H}$ that contains $\mathcal{A}-\mathcal{B}$ and also misses the origin, in which case we may simply take $\mathcal{F}=\mathcal{A}-\mathcal{B}$. So we need only consider the case where the origin lies within the affine hull of $\mathcal{A}-\mathcal{B}$, which we may assume is $\mathbb{R}^{n}$. This allows us to write

$$
\mathcal{A}-\mathcal{B}=\left\{\mathbf{x} \in \mathbb{R}^{n} \mid \mathbf{G}^{T} \mathbf{x} \leq \mathbf{g}\right\}
$$


where each constraint $\mathbf{G}_{j}^{T} \mathbf{x} \leq \mathbf{g}_{j}$ defines an $(n-1)$-dimensional face of $\mathcal{A}-\mathcal{B}$. Because $\mathcal{A}-\mathcal{B}$ has full dimension in $\mathbb{R}^{n}$, there exists some $\hat{\mathbf{x}} \in \mathcal{A}-\mathcal{B}$ with $\mathbf{G}^{T} \hat{\mathbf{x}}<\mathbf{g}$. The proper separation of the origin from $\mathcal{A}-\mathcal{B}$ therefore implies that $\mathbf{g}_{j} \geq 0$ for some index $j$. Defining

$$
\mathcal{H}=\left\{\mathbf{x} \mid \mathbf{G}_{j}^{T} \mathbf{x}=\mathbf{g}_{j}\right\}, \quad \mathcal{F}=\mathcal{H} \cap(\mathcal{A}-\mathcal{B}),
$$

we see that $\mathcal{F}$ is an $(n-1)$-dimensional face of $\mathcal{A}-\mathcal{B}$ contained in the hyperplane $\mathcal{H}$ properly separating the origin from $\mathcal{A}-\mathcal{B}$, as desired.

We will use $\mathcal{H}$ and $\mathcal{F}$ to define faces and separating hyperplanes for $\mathcal{A}$ and $\mathcal{B}$. Note that $\mathcal{H}$ and $\mathcal{F}$ admit representations $\mathcal{H}=\{\mathbf{z} \mid \mathbf{h} \cdot \mathbf{z}=\delta\}$ and

$$
\mathcal{F}=\underset{\mathbf{z}}{\operatorname{argmax}}\{\mathbf{h} \cdot \mathbf{z} \mid \mathbf{z} \in \mathcal{A}-\mathcal{B}\}=\underset{(\mathbf{x}, \mathbf{y})}{\operatorname{argmax}}\{\mathbf{h} \cdot(\mathbf{x}-\mathbf{y}) \mid \mathbf{x} \in \mathcal{A}, \mathbf{y} \in \mathcal{B}\},
$$

where $\delta=\max _{\mathbf{z}}\{\mathbf{h} \cdot \mathbf{z} \mid \mathbf{z} \in \mathcal{A}-\mathcal{B}\}$ and $0 \geq \delta>\inf _{\mathbf{z}}\{\mathbf{h} \cdot \mathbf{z} \mid \mathbf{z} \in \mathcal{A}-\mathcal{B}\}$. In the above representation of $\mathcal{F}$, the maximization over $\mathbf{z}=\mathbf{x}-\mathbf{y}$ can be carried out separately in $\mathbf{x} \in \mathcal{A}$ and $\mathbf{y} \in \mathcal{B}$. This leads us to define

$$
\alpha=\max _{\mathbf{x}}\{\mathbf{h} \cdot \mathbf{x} \mid \mathbf{x} \in \mathcal{A}\}, \quad \beta=\max _{\mathbf{y}}\{-\mathbf{h} \cdot \mathbf{y} \mid \mathbf{y} \in \mathcal{B}\},
$$

which satisfy $\alpha+\beta=\delta \leq 0$. Next, consider the sets

$$
\begin{array}{ll}
\mathcal{H}_{\mathcal{A}}=\{\mathbf{x} \mid \mathbf{h} \cdot \mathbf{x}=\alpha\}, & \mathcal{F}_{\mathcal{A}}=\underset{\mathbf{x}}{\operatorname{argmax}}\{\mathbf{h} \cdot \mathbf{x} \mid \mathbf{x} \in \mathcal{A}\}, \\
\mathcal{H}_{\mathcal{B}}=\{\mathbf{y} \mid-\mathbf{h} \cdot \mathbf{y}=\beta\}, & \mathcal{F}_{\mathcal{B}}=\underset{\mathbf{y}}{\operatorname{argmax}}\{-\mathbf{h} \cdot \mathbf{y} \mid \mathbf{y} \in \mathcal{B}\} .
\end{array}
$$

It is clear that $\mathcal{F}=\mathcal{F}_{\mathcal{A}}-\mathcal{F}_{\mathcal{B}}$, that $\mathcal{H}_{\mathcal{A}}$ and $\mathcal{H}_{\mathcal{B}}$ are hyperplanes properly separating $\mathcal{A}$ and $\mathcal{B}$, and that $\mathcal{F}_{\mathcal{A}}=\mathcal{H}_{\mathcal{A}} \cap \mathcal{A}$ and $\mathcal{F}_{\mathcal{B}}=\mathcal{H}_{\mathcal{B}} \cap \mathcal{B}$ are faces of $\mathcal{A}$ and $\mathcal{B}$ (respectively). Furthermore, the dimensions of these faces satisfy

$$
\operatorname{dim} \mathcal{F}_{\mathcal{A}}+\operatorname{dim} \mathcal{F}_{\mathcal{B}} \geq \operatorname{dim}\left(\mathcal{F}_{\mathcal{A}}-\mathcal{F}_{\mathcal{B}}\right)=\operatorname{dim}(\mathcal{A}-\mathcal{B})-1=\operatorname{dim}(\mathcal{A} \cup \mathcal{B})-1
$$

We have therefore proved the following result.

Theorem 2 Consider nonempty convex polyhedra $\mathcal{A}$ and $\mathcal{B}$ in an Euclidean space and suppose that $\mathcal{A}$ and $\mathcal{B}$ can be properly separated. Then there exist parallel hyperplanes $\mathcal{H}_{\mathcal{A}}$ and $\mathcal{H}_{\mathcal{B}}$, properly separating $\mathcal{A}$ and $\mathcal{B}$, for which $\operatorname{dim}\left(\mathcal{H}_{\mathcal{A}} \cap \mathcal{A}\right)+$ $\operatorname{dim}\left(\mathcal{H}_{\mathcal{B}} \cap \mathcal{B}\right) \geq \operatorname{dim}(\mathcal{A} \cup \mathcal{B})-1$.

Now define $d=\max \{\operatorname{dim} \mathcal{A}, \operatorname{dim} \mathcal{B}\} \leq \operatorname{dim}(\mathcal{A} \cup \mathcal{B})$. To complete the proof of Theorem 1 , we simply note that inequality (1) implies

$$
\max \left\{\operatorname{dim} \mathcal{F}_{\mathcal{A}}, \operatorname{dim} \mathcal{F}_{\mathcal{B}}\right\} \geq \frac{\operatorname{dim} \mathcal{F}_{\mathcal{A}}+\operatorname{dim} \mathcal{F}_{\mathcal{B}}}{2} \geq \frac{d-1}{2} \geq\lfloor d / 2\rfloor-(1 / 2),
$$

so either $\mathcal{F}_{\mathcal{A}}$ or $\mathcal{F}_{\mathcal{B}}$ has dimension at least $\lfloor d / 2\rfloor$. 
A result similar to Theorem 2 for strongly separated convex polyhedra (i.e., those with disjoint closures) is stated without proof or reference in an unpublished technical report [3] by Klee in 1969. This suggests that the some of the ideas considered in the present work have been studied previously, but gives no indication of the context or methods involved in such earlier work.

\section{Examples Showing Optimality of the Bound}

We now present an example in which the two polyhedra meet only at a common vertex. Consider $\mathcal{A}=\mathbb{R}_{+}^{d}$ and $\mathcal{B}=\mathbf{M}\left(\mathbb{R}_{+}^{d}\right)$, where $\mathbf{M}$ is the orthogonal reflection matrix $\mathbf{M}=\mathbf{I}-(2 / d) \mathbf{e e}^{T}$. In dimension $d=3$, the set $\mathcal{B}$ amounts to a copy of the nonpositive octant that has been rotated about the line spanned by the vector $\mathbf{e}$. The rotation puts the edges of $\mathcal{B}$ "opposite" the faces of the nonnegative octant $\mathcal{A}$ in such a way that no face of either polyhedron can be extended to a hyperplane without intersecting the other's interior.

To formalize the preceding statement, consider a hyperplane $\mathcal{H}$ whose nonempty intersection with $\mathcal{A}$ is a $k$-dimensional face of $\mathcal{A}$; note that $\mathcal{H}$ is necessarily a linear subspace of $\mathbb{R}^{d}$. We will prove that $\mathcal{H}$ meets the interior of $\mathcal{B}$ whenever $k>d / 2$; as the roles of $\mathcal{A}$ and $\mathcal{B}$ can be reversed under the isometry $\mathbf{x} \mapsto \mathbf{M x}$, this verifies the optimality of the bound in Theorem 1. The minimal generating set for the cone $\mathcal{A}$ consists of the columns of the identity matrix, whereas the minimal generating set for the cone $\mathcal{B}$ consists of the columns of $\mathbf{M}$. Furthermore, there is a one-toone correspondence between the faces of each cone and the subsets of its generating set, with the face dimension equaling the number of generators in the corresponding subset. Let $\mathbf{e}^{(j)}$ denote the $j$ th column of the identity matrix and define $K=$ $\left\{j \mid \mathbf{e}^{(j)} \in \mathcal{H}\right\}$, so that $K$ lists the generators in the face $\mathcal{H} \cap \mathcal{A}$ of $\mathcal{A}$ and $|K|=k$. The vector

$$
\mathbf{x}=\sum_{j \in K} \mathbf{e}^{(j)}
$$

also belongs to that face, and so $-\mathbf{x} \in \mathcal{H}$. It remains to show that $-\mathbf{x} \in \operatorname{int} \mathcal{B}$, or equivalently, that all entries in the vector $\mathbf{y}=\mathbf{M}^{-1}(-\mathbf{x})$ are positive. This calculation is straightforward:

$$
\mathbf{y}=\mathbf{M}^{-1}(-\mathbf{x})=-\mathbf{M}^{-1} \mathbf{x}=\frac{2}{d} \mathbf{e}(\mathbf{e} \cdot \mathbf{x})-\mathbf{x}=\frac{2 k}{d} \mathbf{e}-\sum_{j \in K} \mathbf{e}^{(j)},
$$

so the entries of $\mathbf{y}$ are given by

$$
\mathbf{y}_{j}= \begin{cases}\frac{2 k}{d}-1 & \text { if } j \in K, \\ \frac{2 k}{d} & \text { if } j \notin K .\end{cases}
$$

Thus $\mathbf{y}_{j}>0$ for all $j$ if $k>d / 2$, which completes the example.

The above example is readily modified so that $\mathcal{A}$ and $\mathcal{B}$ are disjoint: simply translate $\mathcal{B}$ along the direction of $-\mathbf{e}$ by a distance small enough to guarantee that $\operatorname{int}(-\mathcal{B})$ 
still contains all of the finitely many vectors $\mathbf{x}$ of the form (2). Then the analysis of the preceding paragraph still applies.

Next we give an example in which the separating hyperplane is unique and the polyhedra intersect in a singleton that is a vertex of neither polyhedron. We define these polyhedra by

$$
\begin{aligned}
& \mathcal{A}=\left\{\mathbf{x} \in \mathbb{R}^{d} \mid-\left[\begin{array}{ccc}
\mathbf{I}^{(k)} & \mathbf{0} & \mathbf{e} \\
-\mathbf{I}^{(k)} & \mathbf{0} & \mathbf{e}
\end{array}\right] \mathbf{x} \leq \mathbf{0}\right\}, \\
& \mathcal{B}=\left\{\mathbf{x} \in \mathbb{R}^{d} \mid\left[\begin{array}{ccc}
\mathbf{0} & \mathbf{I}^{(d-k-1)} & \mathbf{e} \\
\mathbf{0} & -\mathbf{I}^{(d-k-1)} & \mathbf{e}
\end{array}\right] \mathbf{x} \leq \mathbf{0}\right\},
\end{aligned}
$$

where $k=\lfloor d / 2\rfloor$, and $\mathbf{I}^{(p)}$ denotes the $p \times p$ identity matrix. Visually, one should imagine two solid cubes whose intersection is an edge of both, and then rotate one of the cubes about the midpoint of that edge. The sets $\mathcal{A}$ and $\mathcal{B}$ defined here amount to extending each cube infinitely along its tangent directions by rays from that midpoint, giving objects with a simpler algebraic representation. It is readily verified that $\mathbf{e}^{(d)} \in$ int $\mathcal{A} \cap(-$ int $\mathcal{B})$ and $\mathcal{A} \cap \mathcal{B}=\{\mathbf{0}\}$, that the unique separating hyperplane passes through the origin and is normal to $\mathrm{e}^{(d)}$, and that the maximal faces of $\mathcal{A}$ and $\mathcal{B}$ contained in this hyperplane are the orthogonal subspaces spanned, respectively, by $\left\{\mathbf{e}^{(1)}, \ldots, \mathbf{e}^{(k)}\right\}$ and $\left\{\mathbf{e}^{(k+1)}, \ldots, \mathbf{e}^{(d-1)}\right\}$. This example can be modified slightly by translating $\mathcal{A}$ in the direction of $\mathrm{e}^{(d)}$, thereby giving an example in which $\mathcal{A}$ and $\mathcal{B}$ are disjoint and the maximal faces (among those contained in a separating hyperplane) are uniquely determined within $\mathcal{A}$ and $\mathcal{B}$.

\section{Calculating the Supporting Hyperplanes}

In this section, we demonstrate how linear programming techniques can be employed to find the supporting hyperplanes of Theorem 2 when the polyhedra $\mathcal{A}$ and $\mathcal{B}$ are represented as finite systems of weak linear inequalities. It is well known that linear programs with rational data are solvable in polynomial time [5] and that real-number algorithms for linear programming are finitely convergent.

We first consider the important special case where one of the two polyhedra is known in advance to have nonempty interior. The general case can be handled similarly but requires some additional considerations: we briefly indicate what is needed after treating the full-dimensional case.

\subsection{Hyperplanes for the Full-Dimensional Case}

We now consider the special case where one of the two polyhedra is known to have nonempty interior, in which case we formulate the problem of finding the supporting hyperplanes of Theorem 2 as a dual pair of explicit linear programming problems.

Consider two nonempty closed convex polyhedra $\mathcal{A}, \mathcal{B} \subseteq \mathbb{R}^{d}$ represented as

$$
\mathcal{A}=\left\{\mathbf{x} \in \mathbb{R}^{d}: \mathbf{A}^{T} \mathbf{x} \leq \mathbf{a}\right\}, \quad \mathcal{B}=\left\{\mathbf{x} \in \mathbb{R}^{d}: \mathbf{B}^{T} \mathbf{x} \leq \mathbf{b}\right\},
$$


for some choice of (real) $d \times m$ matrix $\mathbf{A}, d \times n$ matrix $\mathbf{B}, m$-vector $\mathbf{a}$, and $n$-vector $\mathbf{b}$. We shall assume throughout this subsection that the interior of $\mathcal{A}$ is nonempty and that the matrix $\mathbf{A}$ has no zero columns. Hence, the interior of the set $\mathcal{A}$ corresponds to

$$
\operatorname{int} \mathcal{A}=\left\{\mathbf{x} \in \mathbb{R}^{d}: \mathbf{A}^{T} \mathbf{x}<\mathbf{a}\right\}=\bigcup_{\tau>0}\left\{\mathbf{x} \in \mathbb{R}^{d}: \mathbf{A}^{T} \mathbf{x}+\mathbf{e} \tau \leq \mathbf{a}\right\},
$$

where e denotes the vector of all ones. Observe that (int $\mathcal{A}) \cap \mathcal{B}=\emptyset$ if, and only if, the supremum in the linear program

$$
\begin{aligned}
& \text { maximize } \tau \text { over all }(\mathbf{x}, \tau) \in \mathbb{R}^{d} \times \mathbb{R} \\
& \text { subject to } \mathbf{A}^{T} \mathbf{x}+\mathbf{e} \tau \leq \mathbf{a}, \mathbf{B}^{T} \mathbf{x} \leq \mathbf{b},
\end{aligned}
$$

is nonpositive. Also note that any $\mathbf{x} \in \mathcal{B}$ yields a feasible point for (3) by choosing $\tau \leq 0$ with $|\tau|$ sufficiently large that $\mathbf{e} \tau \leq \mathbf{a}-\mathbf{A}^{T} \mathbf{x}$.

For the remainder of this subsection, we assume that (int $\mathcal{A}) \cap \mathcal{B}=\emptyset$, so that problem (3) admits an optimal solution $(\overline{\mathbf{x}}, \bar{\tau})$ with $\bar{\tau} \leq 0$. By linear programming duality, the dual problem

$$
\begin{aligned}
& \operatorname{minimize} \mathbf{a} \cdot \mathbf{u}+\mathbf{b} \cdot \mathbf{v} \text { over all }(\mathbf{u}, \mathbf{v}) \in \mathbb{R}^{m} \times \mathbb{R}^{n} \\
& \text { subject to } \mathbf{A u}+\mathbf{B v}=\mathbf{0}, \mathbf{e} \cdot \mathbf{u}=1, \mathbf{u} \geq \mathbf{0}, \mathbf{v} \geq \mathbf{0},
\end{aligned}
$$

must admit an optimal basic solution $(\overline{\mathbf{u}}, \overline{\mathbf{v}})$, and the optimal values for problems (3) and (4) must be equal: $\bar{\tau}=\mathbf{a} \cdot \overline{\mathbf{u}}+\mathbf{b} \cdot \overline{\mathbf{v}} \leq 0$. We use the dual solution $(\overline{\mathbf{u}}, \overline{\mathbf{v}})$ to define affine sets

$$
\mathcal{H}_{\mathcal{A}}=\left\{\mathbf{x} \in \mathbb{R}^{d}:(\mathbf{A} \overline{\mathbf{u}}) \cdot \mathbf{x}=\alpha\right\}, \quad \mathcal{H}_{\mathcal{B}}=\left\{\mathbf{x} \in \mathbb{R}^{d}:(\mathbf{B} \overline{\mathbf{v}}) \cdot \mathbf{x}=\beta\right\},
$$

where the scalars $\alpha$ and $\beta$ are given by

$$
\alpha=\max _{\mathbf{x}}\left\{(\mathbf{A} \overline{\mathbf{u}}) \cdot \mathbf{x}: \mathbf{A}^{T} \mathbf{x} \leq \mathbf{a}\right\}, \quad \beta=\max _{\mathbf{x}}\left\{(\mathbf{B} \overline{\mathbf{v}}) \cdot \mathbf{x}: \mathbf{B}^{T} \mathbf{x} \leq \mathbf{b}\right\} .
$$

The argument from here is summarized in the following three lemmas.

Lemma 1 The sets $\mathcal{H}_{\mathcal{A}}$ and $\mathcal{H}_{\mathcal{B}}$ defined above are hyperplanes separating $\mathcal{A}$ and $\mathcal{B}$, and the sets $\mathcal{F}_{\mathcal{A}}=\mathcal{H}_{\mathcal{A}} \cap \mathcal{A}$ and $\mathcal{F}_{\mathcal{B}}=\mathcal{H}_{\mathcal{B}} \cap \mathcal{B}$ are nonempty faces of $\mathcal{A}$ and $\mathcal{B}$, respectively.

Proof First note that, by linear programming duality, we have

$$
\begin{aligned}
& \alpha=\max _{\mathbf{x}}\left\{(\mathbf{A} \overline{\mathbf{u}}) \cdot \mathbf{x}: \mathbf{A}^{T} \mathbf{x} \leq \mathbf{a}\right\}=\min _{\mathbf{u}}\{\mathbf{a} \cdot \mathbf{u}: \mathbf{A u}=\mathbf{A} \overline{\mathbf{u}}, \mathbf{u} \geq \mathbf{0}\} \leq \mathbf{a} \cdot \overline{\mathbf{u}}, \\
& \beta=\max _{\mathbf{x}}\left\{(\mathbf{B} \overline{\mathbf{v}}) \cdot \mathbf{x}: \mathbf{B}^{T} \mathbf{x} \leq \mathbf{b}\right\}=\min _{\mathbf{v}}\{\mathbf{b} \cdot \mathbf{v}: \mathbf{B v}=\mathbf{B} \overline{\mathbf{v}}, \mathbf{v} \geq \mathbf{0}\} \leq \mathbf{b} \cdot \overline{\mathbf{v}} .
\end{aligned}
$$

This shows that $\alpha$ and $\beta$ are finite, so $\mathcal{H}_{\mathcal{A}} \cap \mathcal{A}$ and $\mathcal{H}_{\mathcal{B}} \cap \mathcal{B}$ are nonempty. To verify that $\mathcal{H}_{\mathcal{A}}$ and $\mathcal{H}_{\mathcal{B}}$ are hyperplanes, we must show that the vector $\mathbf{A} \overline{\mathbf{u}}=-\mathbf{B} \overline{\mathbf{v}}$ is nonzero. Suppose instead that $\mathbf{A} \overline{\mathbf{u}}=\mathbf{0}$. Then $\alpha=\beta=0$, so we have $\mathbf{a} \cdot \overline{\mathbf{u}} \geq 0$ and 
$\mathbf{b} \cdot \overline{\mathbf{v}} \geq 0$. This implies that $\mathbf{a} \cdot \overline{\mathbf{u}}=0=\mathbf{b} \cdot \overline{\mathbf{v}}$, because $0 \geq \bar{\tau}=\mathbf{a} \cdot \overline{\mathbf{u}}+\mathbf{b} \cdot \overline{\mathbf{v}}$. By the constraint $\mathbf{e} \cdot \mathbf{u}=1$ in (4), we see that $\overline{\mathbf{u}}$ is nonzero. For an interior point $\hat{\mathbf{x}}$ of $\mathcal{A}$, we have $\mathbf{A}^{T} \hat{\mathbf{x}}<\mathbf{a}$, so that

$$
0=\mathbf{0} \cdot \overline{\mathbf{u}}<\left(\mathbf{a}-\mathbf{A}^{T} \hat{\mathbf{x}}\right) \cdot \overline{\mathbf{u}}=\mathbf{a} \cdot \overline{\mathbf{u}}=0 .
$$

This is a contradiction, which proves that $\mathbf{A} \overline{\mathbf{u}}=-\mathbf{B} \overline{\mathbf{v}}$ must be nonzero after all. It remains to demonstrate that both hyperplanes separate $\mathcal{A}$ and $\mathcal{B}$. The optimality of $(\overline{\mathbf{u}}, \overline{\mathbf{v}})$ in problem (4) gives us

$$
\mathbf{A}^{T} \mathbf{x} \leq \mathbf{a} \Longrightarrow \overline{\mathbf{u}} \cdot \mathbf{A}^{T} \mathbf{x} \leq \overline{\mathbf{u}} \cdot \mathbf{a} \Longrightarrow-\overline{\mathbf{v}} \cdot \mathbf{B}^{T} \mathbf{x} \leq-\overline{\mathbf{v}} \cdot \mathbf{b} \leq-\beta .
$$

In other words, each $\mathbf{x} \in \mathcal{A}$ satisfies $(\mathbf{B} \overline{\mathbf{v}}) \cdot \mathbf{x} \geq \beta$, whereas each $\mathbf{x} \in \mathcal{B}$ clearly satisfies the reverse inequality. Thus, the hyperplane $\mathcal{H}_{\mathcal{B}}$ separates $\mathcal{A}$ and $\mathcal{B}$. A similar argument shows that $\mathcal{H}_{\mathcal{A}}$ also separates $\mathcal{A}$ and $\mathcal{B}$.

In the next two lemmas, let $\mathbf{A}_{U}$ denote the submatrix of $\mathbf{A}$ consisting of all columns $\mathbf{A}_{j}$ for which $\overline{\mathbf{u}}_{j}>0$; similarly, let $\mathbf{B}_{V}$ denote the submatrix of $\mathbf{B}$ consisting of all columns $\mathbf{B}_{j}$ for which $\overline{\mathbf{v}}_{j}>0$.

Lemma 2 The dimensions of the faces $\mathcal{F}_{\mathcal{A}}$ and $\mathcal{F}_{\mathcal{B}}$ satisfy

$$
\operatorname{dim}\left(\mathcal{F}_{\mathcal{A}}\right) \geq d-\operatorname{rank}\left(\mathbf{A}_{U}\right), \quad \operatorname{dim}\left(\mathcal{F}_{\mathcal{B}}\right) \geq d-\operatorname{rank}\left(\mathbf{B}_{V}\right) .
$$

Proof We may express the face $\mathcal{F}_{\mathcal{A}}=\mathcal{H}_{\mathcal{A}} \cap \mathcal{A}$ as

$$
\mathcal{H}_{\mathcal{A}} \cap \mathcal{A}=\left\{\mathbf{x} \in \mathbb{R}^{d}:\left(\mathbf{A}_{U} \overline{\mathbf{u}}_{U}\right)^{T} \mathbf{x}=\alpha, \mathbf{A}_{K}^{T} \mathbf{x}=\mathbf{a}_{K}, \mathbf{A}_{J}^{T} \mathbf{x} \leq \mathbf{a}_{J}\right\}
$$

where the column indices of $\mathbf{A}$ are partitioned into sets

$$
\begin{aligned}
& J=\left\{j: \mathbf{A}_{j}^{T} \mathbf{x}<\mathbf{a}_{j} \text { for some } \mathbf{x} \in \mathcal{H}_{\mathcal{A}} \cap \mathcal{A}\right\}, \\
& K=\left\{j: \mathbf{A}_{j}^{T} \mathbf{x}=\mathbf{a}_{j} \text { for all } \mathbf{x} \in \mathcal{H}_{\mathcal{A}} \cap \mathcal{A}\right\} .
\end{aligned}
$$

This shows that $\operatorname{dim}\left(\mathcal{H}_{\mathcal{A}} \cap \mathcal{A}\right)=\operatorname{dim}(\mathcal{L})$, where

$$
\begin{aligned}
\mathcal{L} & =\left\{\mathbf{x} \in \mathbb{R}^{d}:\left(\mathbf{A}_{U} \overline{\mathbf{u}}_{U}\right)^{T} \mathbf{x}=0, \mathbf{A}_{K}^{T} \mathbf{x}=\mathbf{0}\right\} \\
& =\left\{\mathbf{x} \in \mathbb{R}^{d}:\left(\mathbf{A}_{U} \overline{\mathbf{u}}_{U}\right)^{T} \mathbf{x}=0, \mathbf{A}_{K}^{T} \mathbf{x} \leq \mathbf{0}\right\}
\end{aligned}
$$

Without loss of generality, we may assume that $K \backslash U=\{1, \ldots, k\}$. We will inductively construct matrices $\mathbf{G}^{(k)}, \mathbf{G}^{(k-1)}, \ldots, \mathbf{G}^{(0)}$ satisfying

$$
\mathcal{L}=\left\{\mathbf{x} \in \mathbb{R}^{d} \mid\left(\mathbf{A}_{U} \mathbf{G}^{(i)}\right)^{T} \mathbf{x}=\mathbf{0}, \mathbf{A}_{j}^{T} \mathbf{x}=0(\text { for } j=1, \ldots, i)\right\}
$$

for each $i=k, k-1, \ldots, 0$. For the base step $i=k$, we take $\mathbf{G}^{(k)}=\overline{\mathbf{u}}_{U}$, which corresponds to the representation (6). For the inductive step, suppose that the representation (7) holds for an index $i \in K$. We claim that $\mathbf{A}_{i} \in \operatorname{range}\left[\mathbf{A}_{I}, \mathbf{A}_{U} \mathbf{G}^{(i)}\right]$, where we define $I=\{1, \ldots, i-1\}$. If this were not the case, then the Fredholm alternative 
would guarantee the existence of a vector $\mathbf{z}$ satisfying both $\left[\mathbf{A}_{I}, \mathbf{A}_{U} \mathbf{G}^{(i)}\right]^{T} \mathbf{z}=\mathbf{0}$ and $\mathbf{A}_{i}^{T} \mathbf{z}=-1<0$, which contradicts the assumption that $i \in K$. Consequently, we must have $\mathbf{A}_{i}=\mathbf{A}_{I} \mathbf{p}+\mathbf{A}_{U} \mathbf{q}$ for some choice of vectors $\mathbf{p}$ and $\mathbf{q}$, which allows us to replace the equation $\mathbf{A}_{i}^{T} \mathbf{x}=0$ with the equation $\left(\mathbf{A}_{U} \mathbf{q}\right)^{T} \mathbf{x}=0$. In other words, we may take $\mathbf{G}^{(i-1)}=\left[\mathbf{G}^{(i)}, \mathbf{q}\right]$, thereby completing the inductive step. The procedure continues until $i=0$, at which point (7) simply states that $\mathcal{L}=\operatorname{null}\left(\mathbf{A}_{U} \mathbf{G}^{(0)}\right)^{T}$. This implies that $\operatorname{dim}\left(\mathcal{H}_{\mathcal{A}} \cap \mathcal{A}\right)=\operatorname{dim}(\mathcal{L})=d-\operatorname{rank}\left(\mathbf{A}_{U} \mathbf{G}^{(0)}\right) \geq d-\operatorname{rank}\left(\mathbf{A}_{U}\right)$, as desired. The bound for $\operatorname{dim}\left(\mathcal{H}_{\mathcal{B}} \cap \mathcal{B}\right)$ is proved the same way.

Lemma 3 One has $d+1 \geq \operatorname{rank}\left(\mathbf{A}_{U}\right)+\operatorname{rank}\left(\mathbf{B}_{V}\right)$.

Proof There are $d+1$ equality constraints in the dual program (4), so the basic feasible solution $(\overline{\mathbf{u}}, \overline{\mathbf{v}})$ has at most $d+1$ nonzero variables. This can be expressed as $d+1 \geq|U|+|V|$, so it suffices to show that $\operatorname{rank}\left(\mathbf{A}_{U}\right)=|U| \operatorname{and} \operatorname{rank}\left(\mathbf{B}_{V}\right)=|V|$. Because $(\overline{\mathbf{u}}, \overline{\mathbf{v}})$ is basic, the columns of the dual constraint matrix

$$
\mathbf{D}=\left[\begin{array}{cc}
\mathbf{A} & \mathbf{B} \\
\mathbf{e}^{T} & \mathbf{0}
\end{array}\right]
$$

corresponding to the support of $(\overline{\mathbf{u}}, \overline{\mathbf{v}})$ are linearly independent. Clearly, this implies that the columns of $\mathbf{B}_{V}$ are linearly independent, so $\operatorname{rank}\left(\mathbf{B}_{V}\right)=|V|$. Moreover, it also implies the linear independence of the columns of $\mathbf{A}_{U}$. To see why this is true, suppose instead that the columns of $\mathbf{A}_{U}$ are linearly dependent. Then there exists $\hat{\mathbf{u}} \neq \mathbf{0}$ with support $\hat{U} \subseteq U$ such that $\mathbf{A} \hat{\mathbf{u}}=0$. Recall from the proof of Lemma 1 that $\mathbf{A} \overline{\mathbf{u}} \neq \mathbf{0}$, so $\hat{\mathbf{u}}$ cannot be a scalar multiple of $\overline{\mathbf{u}}$. In particular, we must have $\hat{\mathbf{u}}-$ $(\mathbf{e} \cdot \hat{\mathbf{u}}) \overline{\mathbf{u}} \neq \mathbf{0}$. However, we must also have

$$
\mathbf{D}\left[\begin{array}{c}
\hat{\mathbf{u}}-(\mathbf{e} \cdot \hat{\mathbf{u}}) \overline{\mathbf{u}} \\
-(\mathbf{e} \cdot \hat{\mathbf{u}}) \overline{\mathbf{v}}
\end{array}\right]=0
$$

which implies that the $(\overline{\mathbf{u}}, \overline{\mathbf{v}})$-supported columns of $\mathbf{D}$ are linearly dependent, a contradiction. Consequently, the rank of $\mathbf{A}_{U}$ equals $|U|$.

Combining Lemmas 2 and 3 yields $\operatorname{dim}\left(\mathcal{F}_{\mathcal{A}}\right)+\operatorname{dim}\left(\mathcal{F}_{\mathcal{B}}\right) \geq d-1$, as desired (cf. Theorem 2). We also note that the parallel hyperplanes $\mathcal{H}_{\mathcal{A}}$ and $\mathcal{H}_{\mathcal{B}}$ are completely defined by the solutions of the problems (3-4) and the maximization in (5) defining $\alpha$; in particular, the optimal vector $\overline{\mathbf{x}}$ of (3) also yields the maximum defining $\beta$ in (5). These observations demonstrate polynomiality in the case of rational input data.

\subsection{Hyperplanes for the General Case}

We now sketch how to handle the situation in which (possibly) both polyhedra have empty interiors. Given any polyhedron expressed as a system of weak linear inequalities, one can apply standard linear programming techniques to determine which of the inequalities must hold as equations and which hold as strict inequality for at least 
one solution to the system. Consequently, we shall assume in this subsection that the polyhedra $\mathcal{A}, \mathcal{B} \subseteq \mathbb{R}^{d}$ are presented in the form

$$
\mathcal{A}=\left\{\mathbf{x} \in \mathbb{R}^{d}: \mathbf{A}^{T} \mathbf{x} \leq \mathbf{a}, \tilde{\mathbf{A}}^{T} \mathbf{x}=\tilde{\mathbf{a}}\right\}, \quad \mathcal{B}=\left\{\mathbf{x} \in \mathbb{R}^{d}: \mathbf{B}^{T} \mathbf{x} \leq \mathbf{b}, \tilde{\mathbf{B}}^{T} \mathbf{x}=\tilde{\mathbf{b}}\right\},
$$

with their (nonempty) relative interiors given by

$$
\begin{aligned}
& \text { r-int } \mathcal{A}=\left\{\mathbf{x} \in \mathbb{R}^{d}: \mathbf{A}^{T} \mathbf{x}<\mathbf{a}, \tilde{\mathbf{A}}^{T} \mathbf{x}=\tilde{\mathbf{a}}\right\}, \\
& \text { r-int } \mathcal{B}=\left\{\mathbf{x} \in \mathbb{R}^{d}: \mathbf{B}^{T} \mathbf{x}<\mathbf{b}, \tilde{\mathbf{B}}^{T} \mathbf{x}=\tilde{\mathbf{b}}\right\},
\end{aligned}
$$

and their affine hulls expressible as

$$
\operatorname{aff} \mathcal{A}=\left\{\mathbf{x} \in \mathbb{R}^{d}: \tilde{\mathbf{A}}^{T} \mathbf{x}=\tilde{\mathbf{a}}\right\}, \quad \text { aff } \mathcal{B}=\left\{\mathbf{x} \in \mathbb{R}^{d}: \tilde{\mathbf{B}}^{T} \mathbf{x}=\tilde{\mathbf{b}}\right\} .
$$

If either of these affine hulls is the entire space, then the corresponding polyhedron has nonempty interior, and we may simply apply the procedure described in the previous subsection. Otherwise, there are three main cases to consider, depending on the intersections of the above relative interiors and affine hulls. The following describes how to determine which case we are in and how to find the desired hyperplanes $\mathcal{H}_{\mathcal{A}}$ and $\mathcal{H}_{\mathcal{B}}$ for that case.

First, we use linear algebra to determine whether $(\operatorname{aff} \mathcal{A}) \cap(\operatorname{aff} \mathcal{B})=\emptyset$. If this is the case, then we can also use linear algebra to construct parallel hyperplanes $\mathcal{H}_{\mathcal{A}}$ and $\mathcal{H}_{\mathcal{B}}$ strongly separating aff $\mathcal{A}$ from aff $\mathcal{B}$. In particular, we get $\mathcal{F}_{\mathcal{A}}=\mathcal{A}$ and $\mathcal{F}_{\mathcal{B}}=\mathcal{B}$, so we are done.

Otherwise, we determine whether $(\mathrm{r}-\mathrm{int} \mathcal{B}) \cap($ aff $\mathcal{A})=\emptyset$. This is true if, and only if, the supremum in the linear program

$$
\begin{aligned}
& \text { maximize } \sigma \text { over all }(\mathbf{x}, \sigma) \in \mathbb{R}^{d} \times \mathbb{R} \\
& \text { subject to } \tilde{\mathbf{A}}^{T} \mathbf{x}=\tilde{\mathbf{a}}, \mathbf{B}^{T} \mathbf{x}+\mathbf{e} \sigma \leq \mathbf{b}, \tilde{\mathbf{B}}^{T} \mathbf{x}=\tilde{\mathbf{b}}
\end{aligned}
$$

is nonpositive. This problem is feasible because the test in the preceding paragraph told us that $(\operatorname{aff} \mathcal{A}) \cap(\operatorname{aff} \mathcal{B}) \neq \emptyset$. The program dual to $(8)$ is

$$
\begin{aligned}
& \operatorname{minimize} \tilde{\mathbf{a}} \cdot \mathbf{y}+\mathbf{b} \cdot \mathbf{v}+\tilde{\mathbf{b}} \cdot \mathbf{z} \text { over all }(\mathbf{y}, \mathbf{v}, \mathbf{z}) \\
& \text { subject to } \tilde{\mathbf{A}} \mathbf{y}+\mathbf{B v}+\tilde{\mathbf{B}} \mathbf{z}=\mathbf{0}, \mathbf{e} \cdot \mathbf{v}=1, \mathbf{v} \geq \mathbf{0} .
\end{aligned}
$$

If the common optimal value $\bar{\sigma}=\tilde{\mathbf{a}} \cdot \overline{\mathbf{y}}+\mathbf{b} \cdot \overline{\mathbf{v}}+\tilde{\mathbf{b}} \cdot \overline{\mathbf{z}}$ of (8) and (9) is nonpositive, then we can define

$$
\mathcal{H}_{\mathcal{A}}=\left\{\mathbf{x} \in \mathbb{R}^{d}:(\tilde{\mathbf{A}} \overline{\mathbf{y}}) \cdot \mathbf{x}=\alpha\right\}, \quad \mathcal{H}_{\mathcal{B}}=\left\{\mathbf{x} \in \mathbb{R}^{d}:(\mathbf{B} \overline{\mathbf{v}}+\tilde{\mathbf{B}} \overline{\mathbf{z}}) \cdot \mathbf{x}=\beta\right\},
$$

where the scalars $\alpha$ and $\beta$ are given by

$$
\begin{aligned}
& \alpha=\max _{\mathbf{x}}\left\{(\tilde{\mathbf{A}} \overline{\mathbf{y}}) \cdot \mathbf{x}: \tilde{\mathbf{A}}^{T} \mathbf{x}=\tilde{\mathbf{a}}\right\}, \\
& \beta=\max _{\mathbf{x}}\left\{(\mathbf{B} \overline{\mathbf{v}}+\tilde{\mathbf{B}} \overline{\mathbf{z}}) \cdot \mathbf{x}: \mathbf{B}^{T} \mathbf{x} \leq \mathbf{b}, \tilde{\mathbf{B}}^{T} \mathbf{x}=\tilde{\mathbf{b}}\right\} .
\end{aligned}
$$


Lemmas 1, 2, and 3 of Sect. 4.1 can be adapted to show that $\mathcal{H}_{\mathcal{A}}$ and $\mathcal{H}_{\mathcal{B}}$ are hyperplanes satisfying the conclusions of Theorem 2, as desired.

Finally, suppose that the optimal value $\bar{\sigma}$ of the preceding paragraph is positive, so that $(\mathrm{r}$-int $\mathcal{B}) \cap(\operatorname{aff} \mathcal{A}) \neq \emptyset$. In this case, we solve the feasible linear program

$$
\begin{aligned}
& \text { maximize } \tau \text { over all }(\mathbf{x}, \tau) \in \mathbb{R}^{d} \times \mathbb{R} \\
& \text { subject to } \mathbf{A}^{T} \mathbf{x}+\mathbf{e} \tau \leq \mathbf{a}, \tilde{\mathbf{A}}^{T} \mathbf{x}=\tilde{\mathbf{a}}, \mathbf{B}^{T} \mathbf{x} \leq \mathbf{b}, \tilde{\mathbf{B}}^{T} \mathbf{x}=\tilde{\mathbf{b}} .
\end{aligned}
$$

By assumption we have $(\mathrm{r}$-int $\mathcal{B}) \cap(\mathrm{r}$-int $\mathcal{A})=\emptyset$ if, and only if, the supremum in $(10)$ is nonpositive. The dual linear program is

$$
\begin{aligned}
& \operatorname{minimize} \mathbf{a} \cdot \mathbf{u}+\tilde{\mathbf{a}} \cdot \mathbf{y}+\mathbf{b} \cdot \mathbf{v}+\tilde{\mathbf{b}} \cdot \mathbf{z} \text { over all }(\mathbf{u}, \mathbf{y}, \mathbf{v}, \mathbf{z}) \\
& \text { subject to } \mathbf{A u}+\tilde{\mathbf{A}} \mathbf{y}+\mathbf{B v}+\tilde{\mathbf{B}} \mathbf{z}=\mathbf{0}, \mathbf{e} \cdot \mathbf{u}=1, \mathbf{u} \geq \mathbf{0}, \mathbf{v} \geq \mathbf{0} .
\end{aligned}
$$

Assuming that the optimal value $\hat{\tau}=\mathbf{a} \cdot \hat{\mathbf{u}}+\tilde{\mathbf{a}} \cdot \hat{\mathbf{y}}+\mathbf{b} \cdot \hat{\mathbf{v}}+\tilde{\mathbf{b}} \cdot \hat{\mathbf{z}}$ for (10) and (11) is nonpositive, we can define

$$
\mathcal{H}_{\mathcal{A}}=\left\{\mathbf{x} \in \mathbb{R}^{d}:(\mathbf{A} \hat{\mathbf{u}}+\tilde{\mathbf{A}} \hat{\mathbf{y}}) \cdot \mathbf{x}=\alpha\right\}, \quad \mathcal{H}_{\mathcal{B}}=\left\{\mathbf{x} \in \mathbb{R}^{d}:(\mathbf{B} \hat{\mathbf{v}}+\tilde{\mathbf{B}} \hat{\mathbf{z}}) \cdot \mathbf{x}=\beta\right\}
$$

with

$$
\begin{aligned}
& \alpha=\max _{\mathbf{x}}\left\{(\mathbf{A} \hat{\mathbf{u}}+\tilde{\mathbf{A}} \hat{\mathbf{y}}) \cdot \mathbf{x}: \mathbf{A}^{T} \mathbf{x} \leq \mathbf{a}, \tilde{\mathbf{A}}^{T} \mathbf{x}=\tilde{\mathbf{a}}\right\}, \\
& \beta=\max _{\mathbf{x}}\left\{(\mathbf{B} \hat{\mathbf{v}}+\tilde{\mathbf{B}} \hat{\mathbf{z}}) \cdot \mathbf{x}: \mathbf{B}^{T} \mathbf{x} \leq \mathbf{b}, \tilde{\mathbf{B}}^{T} \mathbf{x}=\tilde{\mathbf{b}}\right\}
\end{aligned}
$$

Again, the argument of the preceding subsection can be adapted to show that $\mathcal{H}_{\mathcal{A}}$ and $\mathcal{H}_{\mathcal{B}}$ satisfy the conclusions of Theorem 2 .

\section{References}

1. Anthony, M., Boros, E., Hammer, P.L., Kogan, A. (eds): Discrete Mathematics \& Data Mining. Special issue of Discrete Appl. Math. 144(1-2) (2004)

2. Anthony, M.H.G., Boros, E., Kogan, A., Hammer, P.L. (eds): Discrete Mathematics and Data Mining, II. Special issue of Discrete Appl. Math. 156(6) (2008)

3. Klee, V.: Separation and support properties of convex sets-a survey. Mathematical Note No. 599, D1-82-0842, Boeing Scientific Research Laboratories (1969)

4. Rockafellar, R.T.: Convex Analysis. Princeton University Press, Princeton (1970)

5. Schrijver, A.: Theory of Linear and Integer Programming. Wiley, Chichester (1986) 